\title{
Quality assessment of discharge summaries from an emergency department in eastern Nepal
}

\author{
R Giri, R Bhandari, M Poudel, PP Gupta \\ Department of General Practice and Emergency Medicine \\ B.P. Koirala Institute of Health Sciences, Dharan, Nepal
}

\begin{abstract}
Background: Discharge summaries are intended to transfer important clinical information from inpatient to outpatient settings and between hospital admissions. A good discharge summary helps physician to provide continuity of care which will in turn improve patient outcomes. Despite the importance of the discharge summary, there has been relatively little research in this area in Nepal. We therefore decided to review discharge summaries of patients discharged from emergency department in eastern Nepal. Objective: To assess the completeness of discharge summaries from emergency department. Methods: A total of 360 discharge summaries, representing $20 \%$ of discharge from the emergency department of B.P. Koirala Institute of Health Sciences, Nepal were randomly selected and evaluated. Quality of discharge for completeness was evaluated using recommendations by Joint Commission on Accreditation of Hospital for the presence or absence of the following key items: admission diagnosis, drug allergy, physical examination, significant laboratory test and results, discharge diagnosis, procedures, discharge medication (including dose and duration), follow up and attending physician signature. Results: The proportion of discharge summaries missing particular component of vital data ranged from less than 4\% (no discharge medications) to $97 \%$ (no mention of drug allergy). Information was missing on patients discharge condition (74\%), hospital course (61\%), discharge instruction (57\%) and the discharge diagnosis in (13\%). Most of the discharge summaries were partially structured representing $75 \%$. Ease of access to the diagnosis was $78 \%$. Conclusions: Though most of the discharge summaries were structured and access to the diagnosis was $78 \%$, considerable deficiencies in the completeness of discharge summaries were found.
\end{abstract}

Key word: Discharge, outcome, quality assessment

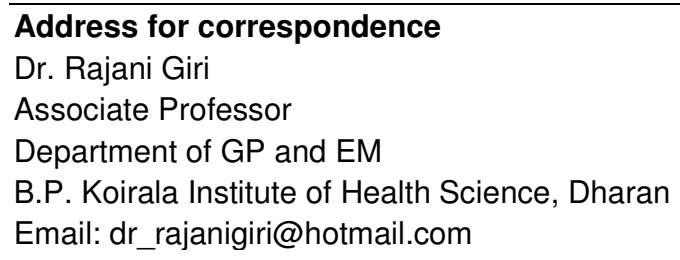




\section{Introduction}

A hospital discharge summary is used to summarize the events of hospitalization. It is a document prepared by the attending physician of a hospitalized patient that summarizes the admitting diagnosis, diagnostic procedures performed, therapy received while hospitalized, clinical course during hospitalization, prognosis, and plan of action upon the patient's discharge with stated time to follow-up. ${ }^{1}$ Van Walraven and Rokosh ${ }^{2}$ proposed a definition for a quality discharge summary: "a high-quality discharge summary efficiently communicates information necessary for ongoing care by a patient's family (primary) physician".

The hospital discharge summary is the most important tool available which is intended to relay important clinical information from inpatient to outpatient settings and between hospital admissions. Often, the discharge summary is the only form of communication that accompanies the patient to the next setting of care. ${ }^{3}$

Lack of information can lead to poor continuity of care, resulting in an unnecessary duplication of consultations or investigations, poly-pharmacy, iatrogenic errors, a worsening of the health condition, patient dissatisfaction, and a subsequent loss of confidence in the medical team and physician ${ }^{4,5}$

Problems with the way discharge summaries are generated have been documented in the medical literature. In one study Stevenson, Boyle and Alexander, noted the unnecessary lengthiness and content deficiencies of discharge summaries. ${ }^{6}$ Studies have shown that as many as forty percent of patients over sixty five had medication errors after leaving the hospital, and eighteen percent of Medicare patients discharged from a hospital are readmitted within thirty days. On the other hand, research has shown that excellent planning and good follow-up can improve patients' health, reduce readmissions and decrease healthcare costs. ${ }^{7}$

Poor discharges can also lead to inefficient care and inappropriate utilization of health care resources. Standardized, consistent discharge summary is one of the key factors in improving the quality of patient care. The joint commission has established standards (standard IM.6.10, EP 7) outlining the components that each hospital discharge summary should contain. ${ }^{8}$ These components are reason for hospitalization, significant findings, procedures and treatment provided, patient's discharge condition, patient and family instructions (as appropriate), attending physician's signature.

We therefore decided to review and assess the completeness of the discharge summary of patients discharged from emergency department of BPKIHS to view the quality of discharge modified from recommendation by Joint Commission on Accreditation. 


\section{Methods}

This was a descriptive retrospective hospital based study, which were carried out in emergency department of BPKIHS Dharan, Nepal. A total of 360 patient representing $20 \%$ of the discharge cases of one month from emergency department was taken. The case sheet from emergency department having discharge summaries, were included.

Patient leaving against medical advice and absconded patients were excluded.

Secondary data from discharge case sheets were taken. Quality of discharge was assessed using case files.

The items included for quality assessment have been determined after a review of literature. Information items on the form included those felt to be important by the researcher for discharge content, modified from recommendation by Joint Commission on Accreditation of Hospital. ${ }^{8}$

Discharge summaries were evaluated for the presence or absence of the following key items: Admission diagnosis, drug allergy, physical examination. Significant laboratory test and results, discharge diagnosis, procedures, discharge medication (including dose and duration,) and medical follow up, attending physician's signature, consultation name.

Measures of the ease of access to important information were also evaluated by judging the structuring of the reports and ease to access to the diagnosis.

Under structuring the report were coded in the following categories: wholly structured defines as being divided completely into marked sections; unstructured which is describe to the essay-style narrative letter in which no data are specially emphasized; or partially structured, defined as the essay style letter with certain important data (specially the diagnosis) highlighted by such means as underlying, the use of block letter or boxing. ${ }^{9}$ Estimation of ease of access to diagnosis depended on whether it was simply part of narrative report or highlighted in some way so that it was clearly identifiable at a glance.

For quality assurance the questionnaire was converted into marking, if the assigned work was performed then the question was given 1 mark otherwise 0 .the scale was categorized to $0,1,2$ for structuring and ease to assess the diagnosis 1,0 . The total mark became 19. If $75 \%$ mark was obtained then it considered of quality.

The discharge were evaluated by investigator and crosschecked by co investigators to omit inter observer bias. The data were directly entered in Microsoft Excel and were analyzed by using SPSS version11. To assess the coefficient of reliability self-modified scale validity test was done where Cron Beck Alfa was 0.68 , so this scale was valid for detecting the quality of paper in emergency. 
Ethical clearance was obtained from ethical board of BPKIHS.

\section{Results}

Total 360 discharge sheet were accessed for completeness of the discharge summaries. The missing item ranged from 4\% (no discharge medication) to $97 \%$ (no mention of drug allergy). Information was missing on patient discharge condition (74\%), hospital course $(61 \%)$ discharge instructions (57\%), HOPI (history of past illness) (53\%). The information on drug allergy was underreported(3.3\%).

Table1: Shows proportion of discharge summaries that were complete or absent

\begin{tabular}{|c|c|c|c|}
\hline Items & Categories & No & $\%$ \\
\hline \multirow{2}{*}{ Admission diagnosis } & Mention & 360 & 100.0 \\
\hline & Not mention & 0 & 0.0 \\
\hline \multirow{2}{*}{ Discharge diagnosis } & Mention & 314 & 87.2 \\
\hline & Not mention & 46 & 12.8 \\
\hline \multirow{2}{*}{ HOPI } & Mention & 168 & 46.7 \\
\hline & Not mention & 192 & 53.2 \\
\hline \multirow[t]{2}{*}{ Drug allergy } & mention & 12 & 3.3 \\
\hline & Not mention & 348 & 96.7 \\
\hline \multirow{2}{*}{ Significant finding } & Mention & 340 & 94.4 \\
\hline & Not mention & 20 & 5.6 \\
\hline \multirow{2}{*}{ Consultation } & Mention & 345 & 95.8 \\
\hline & Not mention & 15 & 4.2 \\
\hline Procedure & $\begin{array}{l}\text { Mention } \\
\text { Not mention }\end{array}$ & 360 & 100 \\
\hline \multirow{2}{*}{ Hospital course } & Mention & 138 & 38.3 \\
\hline & Not mention & 222 & 61.7 \\
\hline \multirow{2}{*}{ Relevant investigation } & Mention & 338 & 93.9 \\
\hline & Not mention & 22 & 6.1 \\
\hline \multirow{2}{*}{ Discharge condition } & Mention & 93 & 25.8 \\
\hline & Not mention & 267 & 74 \\
\hline \multirow{2}{*}{ Discharge medication } & Mention & 345 & 95.8 \\
\hline & Not mention & 15 & 4.2 \\
\hline
\end{tabular}




\begin{tabular}{|c|c|c|c|}
\hline Discharge instruction & $\begin{array}{l}\text { Mention } \\
\text { Not mention }\end{array}$ & $\begin{array}{l}155 \\
205\end{array}$ & $\begin{array}{l}43.1 \\
56.9\end{array}$ \\
\hline Follow up & $\begin{array}{l}\text { Mention } \\
\text { Not mention }\end{array}$ & $\begin{array}{l}314 \\
19\end{array}$ & $\begin{array}{l}94.7 \\
5.3\end{array}$ \\
\hline Attending physician signature & $\begin{array}{l}\text { Mention } \\
\text { Not mention }\end{array}$ & $\begin{array}{l}258 \\
2\end{array}$ & $\begin{array}{l}99.4 \\
0.6\end{array}$ \\
\hline Consultation name & $\begin{array}{l}\text { Mention } \\
\text { Not mention }\end{array}$ & $\begin{array}{l}351 \\
9\end{array}$ & $\begin{array}{l}97.5 \\
2.5\end{array}$ \\
\hline
\end{tabular}

Under structuring, the report was categorized as fully structured, partially structured and unstructured. The table below represents the proportion of structuring of the report and asses to the diagnosis.

Table 2: Structuring of the report and access to the diagnosis

\begin{tabular}{|l|l|l|}
\hline & & \\
\hline & Structuring of the report & \\
Fully structured & 85 & $23 \%$ \\
Partly structured & 270 & $75 \%$ \\
Unstructured & 5 & $1.4 \%$ \\
\hline & & \\
Not Clearly identified clearly identified & 77 & $21.4 \%$ \\
& 283 & $78.6 \%$ \\
\hline
\end{tabular}

Completeness of discharge summaries was assessed using a score. Mean mark obtained was found to be 14.04(SD) +/-2.128 Minimum marked obtained was 6 and maximum was 19 . The table below shows the frequency of the discharge summaries for completeness. Only 87 of the discharge summaries were complete, rest were incomplete.
Table 3: Completeness of discharge summaries

\begin{tabular}{|l|l|}
\hline Mark & Frequency \\
\hline$<10(<50)$ & 5 \\
\hline $10-15(50-75)$ & 268 \\
\hline$>15(75 \% 0$ & 87 \\
\hline
\end{tabular}




\section{Discussions}

Discharge summaries are important documents containing information that is relevant to ongoing patients care and evaluation. Despite its importance well described and the existence of joint commission standards mandating certain discharge summary components, our study found various important component missing in the discharges summaries.

The proportion of DS missing particular vital data ranged from 4\% (no discharge medication) to $97 \%$ (no mention of drugs allergy). Drug allergy was under reported $3.3 \%$ which was similar to the other studies which also found it to be mention less. ${ }^{10}$ This finding is important as it could result in fatal drug adverse effect if not reported. It is likely that it was not mention in the history case sheet also making it mandatory to include drug allergy in discharge format existed in our hospital.

Information was missing on patient discharge condition (74\%), hospital course (61\%) discharge instruction (57\%), HOPI (53\%) in contrast to other studies ${ }^{10}$ which had missing information on admission diagnosis (34\%), discharge diagnosis (25\%), discharge medication (22\%) which was well mentioned in our study. Underreporting of patients discharge condition, hospital course and discharge instruction is of a concern. Lack of good discharge instruction results in polypharmacy and readmission as shown in studies. ${ }^{4,5}$

Incomplete reporting of laboratory tests and results is also a concern as it may cause duplication of the test that has already been performed when patients comes for follow up. A measure of the ease to important information were evaluated by judging the structuring. Structured DS was $23 \%$ which is less comparative to other studies done by $A \mathrm{~J}$ Tulloch ${ }^{9}$ which found to be $58 \%$. The same study found unstructured DS to be $9.5 \%$ which is higher than our study (1.4\%). Remaining $75 \%$ DS in our study were partially structured.

Access to the diagnosis was clearly identifiable at a glance in almost $78 \%$ which is in contrast to other studies $(20 \%)^{9}$. The diagnosis was either written in capital letter or in a box making it visible at a glance. Completeness of DS was found only on 87 case sheets. Five DS was of low quality. Unfortunately the discharge summaries are created by house officers and interns who have minimal training in this area and some missing data may be due to hectic shifts in emergency when they have less time to complete.

\section{Conclusion}

Considerable deficiencies in the completeness of DS were found. Several items could have been recorded better, 
HOPI, hospital course, discharge condition, discharge instruction. Drug allergy was under reported.

Feedback intervention and strategies to improve discharge summaries should be implemented. Supervision by senior consultant is needed to maintain the quality of discharges. Improvement can probably be addressed by introducing electronic medical records if feasible.

\section{References}

1. McGraw-Hill Concise Dictionary of Modern Medicine. The McGraw-Hill Companies;2002. Available at: http://www.thefreedictionary.com/sources. htm. (Accessed Jan 2012)

2. Van Walraven C, E Rokosh. What is necessary for high-quality discharge summaries? Am J Med Qual. 1999; 14(4):160-169.

3. Kripalani S, LeFevre F, Phillips CO, Williams MV, Basaviah P, Baker DW : Deficits in communication and information transfer between hospitalbased and primary care physicians: Implications for patient safety and continuity of care. JAMA 2007; 297: 831841.

4. Piterman L, Koritsas S. Part II. General practitioner-specialist referral process. Intern Med J 2005; 35(8):491-496.
5. Kravitz RL, Reuben DB, Davis JW, Mitchell A, Hemmerling K, Kington RS, et al. Geriatric home assessment after hospital discharge. J Am Geriatr Soc 1994, 42(12):1229-1234.

6. Stevenson JG, Boyle CM, Alexander WD A new hospital discharge letter. Lancet 1973; 1: 928-931.

7. Jeffrey L. Greenwald, MD, Charles R. et al. The Hospital Discharge: A Review of a High Risk Care Transition With Highlights of a Reengineered Discharge Process. Journal of Patient Saf. 2007;2(3).

8. Joint Commission on the Accreditation of Healthcare Organizations. Hospital Accreditation Standards .Standard IM.6.10, EP 7.Available from: http://www.jointcommission.org/NR/rdonly res/A9E4F954-F6B5-4B2D-9ECFC1E792BF390A/0/D_CurrenttoRevised_ DC_HAP.pdf. (accessed February, 2012) 9. Tulloch AJ, Fowler GH, McMullan JJ, Spence JM: Hospital discharge reports: Content and design. Br Med J 1975; 4: 443-446.

10. Van Walraven C, Weinberg AL. Quality assessment of discharge summary system. CMAJ.1995; 152:1437-1442. 